\title{
LA CONSTRUCCIÓN DE LA VOZ DE AUTOR: TRAYECTORIA A TRAVÉS DE UNA TITULACIÓN DE GRADO
}

\author{
Alba Naroa Romero González \\ Manuela de las Nieves Álvarez Álvarez \\ Universidad de Deusto
}

\begin{abstract}
RESUMEN: Este trabajo de investigación presenta un análisis de cómo construyen la voz de autor estudiantes universitarios de una titulación de grado. Desde el enfoque del metadiscurso se plantea como objetivo identificar y analizar los recursos y expresiones utilizadas y trazar la trayectoria a través de los cuatro cursos del programa formativo. Se trata de un estudio de tipo cualitativo que tiene como corpus 96 reseñas producidas por los estudiantes de los cuatro cursos de grado. Los resultados constatan que los informantes configuran una voz de autor neutra que ni valora los contenidos ni resulta persuasiva.
\end{abstract}

PALABRAS CLAVE: Voz de autor, trayectoria formativa, metadiscurso, estudiantes universitarios.

\section{THE CONSTRUCTION OF AUTHORIAL VOICE: TRAJECTORY THROUGHOUT AN UNIVERSITY DEGREE}

\begin{abstract}
This research presents an analysis of how university students of a specific degree constructs their authorial voice. Using metadiscourse approach, the objectives are to identify and analyse resources and linguistic expressions they use and to draw their trajectory throughout the four years of the degree. It is a qualitative study based on 96 book reviews written by the four year degree students. The results reveal that the participants set up a neutral authorial voice that not assess the contents exposed and it is not persuasive.
\end{abstract}

KEYWORDS: Authorial voice, academic trajectory, metadiscourse, university students.

Recibido: 04/08/2018

Aceptado: 17/12/2018 
Correspondencia: Alba Naroa Romero González, Facultad de Ciencias Sociales y Humanas, Universidad de Deusto, Avenida de las Universidades, 48007 Bilbao. Email: alromer@opendeusto.es.

\section{INTRODUCCIÓN}

Tradicionalmente, los textos académicos se han caracterizado por la objetividad y la expresión del conocimiento de manera neutra (Castelló, Corcelles, Iñesta, Bañales y Vega, 2011). Esta visión de la voz de autor de textos académicos ha ido cambiando por el empuje de movimientos como el Writing Across the Curriculum (Bazerman, Little, Bethel, Chavkin, Fouquette y Garufis, 2005) y el Academic Literacies (Street, 1999) que han impulsado la investigación sobre la escritura académica desde la consideración del texto como un acto de interacción (Castelló, Corcelles, Iñesta, Bañales y Vega, 2011).

Esta forma de entender la escritura académica requiere de los estudiantes nuevas formas de leer, interpretar y producir los textos que se les solicita porque ya no es suficiente con que reproduzcan el conocimiento, ahora también se valora que se posicionen respectos a los contenidos (Castelló, Corcelles, Iñesta, Bañales y Vega, 2011). Paralelamente, no son pocas los voces (Castro y Sánchez, 2013; Aguilar y Fregoso, 2013) que han constatado que los estudiantes tienen dificultades para construir su propia voz e interactuar con el lector o con otras voces en los textos que producen.

La preocupación por esas dificultades ha generado toda una línea de investigación desde diferentes enfoques y con diferentes objetivos. En este estudio, se sigue el enfoque del metadiscurso propuesto por Hyland (2007), el cual entiende el texto como un medio de comunicación social. Así, el estudio empírico que aquí se presenta se propone, mediante la metodología de análisis del metadiscurso, analizar cómo construyen los estudiantes de toda una titulación de grado la voz del autor en reseñas producidas por ellos. Este objetivo general se concreta en los siguientes objetivos específicos: (1) identificar y analizar los recursos y expresiones utilizados por los estudiantes en la reseña para presentarse a sí mismos en el texto, para posicionarse respecto a los contenidos, para interactuar con el lector y para interactuar con otras voces; (2) analizar y caracterizar la trayectoria en la construcción de la voz de autor en el transcurso de una titulación de grado.

\section{El METADISCURSO}

Los modos que tiene el autor de crear su propia voz en el discurso recibe diferentes aportes teóricos procedentes de la Escuela Francesa de Análisis del Discurso (Benveniste, 1974), los estudios sobre la Despersonalización o Desagentivación del discurso (Goethals y Delbecque, 2001), la Modalización del Discurso (Casamiglia y Tusón, 2001), los Estudios de Cortesía y Atenuación (Brown y Levinson, 1987), el Posicionamiento Estratégico del Autor (Meza, 2017) y el Metadiscurso (Hyland, 2005), entre otros.

Entre estos enfoques, el metadiscurso es una destacada corriente de estudio de la voz de autor que aporta una visión completa de este fenómeno al considerar el texto como un medio de comunicación social de carácter situado con objetivos persuasivos. Con ese objeto, integra todo un conjunto de aspectos discursivos en un mismo análisis que desde otras orientaciones analíticas se tratan de manera aislada. 
Existen diferentes propuestas del metadiscurso entre las que destacan las de VandeKoople (1985), Crismore, Markkanen y Steffensen (1993) y Hyland (2005). El primero de estos autores, Vande-Koople (1985), hace una propuesta en la que el metadiscurso (interpersonal y textual) está al servicio del contenido ideacional en el sentido en que refleja la actitud, el posicionamiento e incluso la evaluación de los contenidos del texto por parte del autor. Más tarde, Crismore, Markkanen y Steffensen (1993) partiendo de la propuesta de Vande-Koople, eliminan, crean y reformulan algunas categorías con el fin de evitar las ambigüedades detectadas en el modelo anterior.

Ambas propuestas son criticadas debido al enfoque. Aunque ambas se basan en un enfoque funcional, las categorías de análisis que proponen siguen, por el contrario, criterios sintácticos. Además, algunas categorías resultan excesivamente ambiguas, lo que produce confusiones en el análisis hasta tal punto que, en la clasificación de Crismore et al. (1985), no es clara la división del metadiscurso en la función textual y la función interpretativa (Hyland, 2005).

Fruto de estas observaciones, a principios del siglo XXI, Hyland (2005) realiza su propia propuesta en dos dimensiones (interaccional e interactiva), basada en criterios funcionales, que abarca los recursos que utiliza el autor para presentarse a sí mismo en el texto, para posicionarse respecto a los contenidos e interactuar con el lector y con otras voces. Poco después rehará esta misma propuesta, en otro modelo analítico en el que se refiere al metadiscurso interpersonal y el metadiscurso textual (2007). Además de renombrar alguna categoría, no realiza cambios más sustanciales. En la siguiente figura se presenta su propuesta.

\begin{tabular}{|c|c|c|}
\hline Category & Function & Examples \\
\hline & Textual metadiscourse & \\
\hline Logical connectives & $\begin{array}{l}\text { Express semantic relation } \\
\text { between main clauses }\end{array}$ & $\begin{array}{l}\text { in addition / but / thus / } \\
\text { and }\end{array}$ \\
\hline Frame markers & $\begin{array}{l}\text { Explicitly refer to discourse } \\
\text { acts or text stages }\end{array}$ & $\begin{array}{l}\text { finally / to repeat / here } \\
\text { we try to }\end{array}$ \\
\hline Endophoric markers & $\begin{array}{l}\text { Refer to information in other } \\
\text { parts of the text }\end{array}$ & $\begin{array}{l}\text { noted above / see Fig / in } \\
\text { section } 2\end{array}$ \\
\hline Exidentials & $\begin{array}{l}\text { Refer to source of } \\
\text { information from other texts }\end{array}$ & $\begin{array}{l}\text { according to X/(1990)/ } \\
\mathrm{Z} \text { states }\end{array}$ \\
\hline Code glosses & $\begin{array}{l}\text { Help readers grasp meaning } \\
\text { of ideational material } \\
\text { Interpersonal metadiscourse }\end{array}$ & $\begin{array}{l}\text { namely / e.g. / such as / } \\
\text { i.e. }\end{array}$ \\
\hline Hedges & $\begin{array}{l}\text { Withhold writer's full } \\
\text { commitment to statements }\end{array}$ & $\begin{array}{l}\text { might / perhaps / possible } \\
\text { / about }\end{array}$ \\
\hline Boosters & $\begin{array}{l}\text { Emphasize force or writer's } \\
\text { certainty in message }\end{array}$ & $\begin{array}{l}\text { in fact / definitely / It is } \\
\text { clear }\end{array}$ \\
\hline Attitude markers & $\begin{array}{l}\text { Express writer's attitude to } \\
\text { propositional content }\end{array}$ & $\begin{array}{l}\text { Unfortunately / I agree / } \\
\text { X claims }\end{array}$ \\
\hline Relational markers & $\begin{array}{l}\text { Explicitly refer to or build } \\
\text { relationship with reader }\end{array}$ & $\begin{array}{l}\text { frankly / note that / you } \\
\text { can see }\end{array}$ \\
\hline Person markers & $\begin{array}{l}\text { Explicit reference to } \\
\text { author(s) }\end{array}$ & $\mathrm{I} / \mathrm{we} / \mathrm{my} / \mathrm{mine} /$ our \\
\hline
\end{tabular}

Figura 1. Clasificación del metadiscurso modificada propuesta por Hyland (2007, p. 111) 
Pudiera parecer en la lectura de su propuesta que las categorías están perfectamente diferenciadas y que no se pueden producir solapamientos, pero eso depende del uso que realice el autor de ellas (Hyland, 2007). Metadiscurso interpersonal y textual pueden encontrarse en un mismo párrafo, en una misma frase e incluso en una misma palabra; por eso, es tan importante estudiarlo, porque da cuenta de la complejidad del lenguaje y de la importancia de la situación comunicativa (Vande-Koople, 2012).

Conviene, además, tener en cuenta a la hora de analizar un texto mediante el metadiscurso las diferencias que existen en los modos de presentar la voz del autor en las diferentes lenguas (Vande-Koople, 2012). Podría servir de ejemplo el modo que tiene el autor de hacerse presente a sí mismo en el texto en el discurso anglosajón mediante la primera persona del singular (Hyland, 2007) mientras que tiende a desaparecer tras formas verbales en tercera persona en el discurso español (Goethals y Delbecque, 2001). Por esa razón, todo texto analizado mediante el enfoque del metadiscurso debe ser interpretado en el marco de la disciplina, la tradición cultural y el idioma en el que se produce.

\section{El GÉNERO RESEÑA}

El género reseña es categorizado como un género académico desde que, en la década de los noventa, el interés por él dio lugar a toda una línea de investigación (Motta-Roth, 1995; Giannoni, 2000). Se ha argüido que es un género de escasa significatividad para la creación del conocimiento y para el currículum del autor de la reseña (Giannoni, 2000; Alcaraz y Salager-Meyer, 2005), pero, con el paso del tiempo, se ha coronado como un género de gran importancia que facilita al investigador la búsqueda de nuevas fuentes, valida el conocimiento de la disciplina a través de la evaluación y contribuye al intercambio de opiniones entre los académicos de una disciplina (Giannoni, 2009).

No obstante, pese a su importancia y pese a ser uno de los géneros que tanto expertos como estudiantes producen con más frecuencia, la investigación sobre este género sigue siendo muy escasa (Alzari, 2012) frente a otros géneros. Asimismo, los estudios realizados hasta el momento se centran en reseñas producidas por expertos (Giannoni, 2000; Salager-Meyer, 2001; Babaii y Ansary, 2007; Giannoni, 2009; Tse y Hyland, 2009), de manera que todavía se desconoce el comportamiento de los estudiantes en la producción de este tipo de géneros. Se sabe, resultado de la experiencia docente, que, cuando la producen los estudiantes, se caracteriza por ser un texto breve que tiene por objetivo favorecer el aprendizaje de los estudiantes, y que no tiene que ser necesariamente una reseña de una obra de reciente publicación (Alzari, 2012; Alzari, D’Alessandro y Radiminski, 2014).

No son pocas las voces que han subrayado la necesidad y la importancia de que los estudiantes produzcan este género académico (Motta-Roth, 2001; Alzari, D'Alessandro y Radiminski, 2014). Por una parte, han señalado repetidamente que, mediante la producción de reseñas de diferentes géneros acadé- 
micos (ensayos, artículos de investigación, manuales, etc.), los estudiantes se familiarizan con los autores, los temas, la estructura, el registro y desarrollan habilidades para la emisión de opiniones justificadas de otros textos que deben interpretar e, incluso, producir en el futuro (Alzari, D'Alessandro y Radiminski, 2014). Si, además, se propone la producción de reseñas en el marco de diferentes disciplinas, los estudiantes podrán identificar de manera consciente las diferencias disciplinares y, por ende, la diversidad de los discursos en la academia (Motta-Roth, 2001).

Asimismo, aunque la función principal de la reseña es informar sobre novedades bibliográficas relevantes (ya sea por el reconocimiento del autor de la obra, la actualidad del tema o la fecha de publicación) para una disciplina, también tiene otras funciones que requieren del estudiante que analice críticamente la obra y se posicione. Entre estas, según Castro de Castillo (2005), se encuentran (1) expresar juicios de valor, (2) comandar sobre el lector para que lea la obra y (3) contactar con el lector y con otras voces mediante la descripción y la argumentación. Esto requiere del estudiante que sea capaz de construir su propia voz en el texto, de valorar los contenidos críticamente y de adoptar una postura.

\section{Marco metodológico}

\section{Participantes y corpus}

Los participantes de este estudio, por una parte, son los 96 estudiantes que componen la titulación de grado en Lenguas Modernas de la Universidad de Deusto (Bilbao, País Vasco). Todos ellos son estudiantes de entre 17 y 22 años matriculados por primera vez en cada curso, es decir, estudiantes que no han realizado otros estudios universitarios previamente y que no han repetido curso. Todos ellos conforman los cuatro cursos del grado. El corpus, por otra parte, está conformado por 96 reseñas académicas de formación escritas ad hoc, de manera individual, por cada uno de los participantes del estudio.

\section{Instrumento}

Una manera de analizar cómo los estudiantes construyen la voz de autor es examinar una muestra natural de escritura producida a efectos de esta investigación para que todos los textos sean del mismo género, los mismos temas y el mismo nivel de complejidad.

Tras esta consideración inicial, se plantea crear un instrumento que plantee a los estudiantes una situación comunicativa que sea asumible para los estudiantes de todos los cursos del grado y que, además, cumpla con unos requisitos de tiempo, contenido e idoneidad. Desde esta reflexión, se decide que los estudiantes produzcan una reseña académica de formación porque es un género académico, fácilmente reconocible, que requiere de operaciones discursivas y habilidades de selección de los contenidos de cierta complejidad, y cuya instrucción se puede ajustar a todos los niveles del grado. 
Teniendo en cuenta todas estas consideraciones, el primer paso en el diseño del instrumento es la selección del libro objeto de la reseña. Este se selecciona considerando que debe estar en español, debe tratar una temática afín a los estudios de los participantes y que la portada, contraportada, índice y presentación del libro deben ser lo bastante completos para que los informantes cuenten con toda la información que pudieran necesitar. Así es como se selecciona la Gramática didáctica del español de Leonardo Gómez Torrego (1998).

Después, se diseña la consigna. Para ello, se les propone a los estudiantes una situación comunicativa concreta: la biblioteca de la Universidad de Deusto solicita reseñas de los libros de reciente incorporación para su catálogo. Con la única exigencia formal de que la reseña tenga, al menos, 200 palabras, los participantes de este estudio deben producir una reseña que oriente al usuario de la biblioteca en la elección del libro.

Antes de aplicar el instrumento con los estudiantes de Lenguas Modernas, se realiza un pretest con los estudiantes del grado en Lenguas Modernas y Gestión (aquellos cuya formación es más afín a los participantes del estudio). A partir de ese pretest, se realizan correcciones significativas en la consigna que contribuyen a la mejora del instrumento para que responda a los objetivos de la investigación (ver consigna en el Anexo 1).

\section{Procedimiento de análisis}

La caracterización de la voz del autor se realiza mediante el enfoque del metadiscurso de Hyland (2007) por ser el que mejor se ajusta a los objetivos de este estudio. Se analiza cómo se presentan en el texto a sí mismos, cómo se posicionan respecto a los contenidos y cómo interactúan con el lector y con otras voces. Por tanto, se manejarán las categorías del metadiscurso interpersonal y la categoría de evidenciales del metadiscurso textual.

Este análisis de tipo descriptivo se lleva a cabo en el software de Atlas.ti, mediante un procedimiento de constantes lecturas y comparaciones que se fijan en los usos que realizan los estudiantes de los diferentes tipos de metadiscurso, la variedad de recursos y expresiones que utilizan y cómo esos usos cambian a través de su trayectoria formativa.

\section{Resultados Y Discusión}

\section{Corpus global}

El análisis de las 96 reseñas académicas ha permitido constatar la presencia de las cuatro dimensiones planteadas en este estudio: la presencia del autor en el texto, el posicionamiento del autor, la interacción del autor con el lector y la interacción del autor con otras voces. La siguiente figura da muestra de los usos de cada una de estas: 


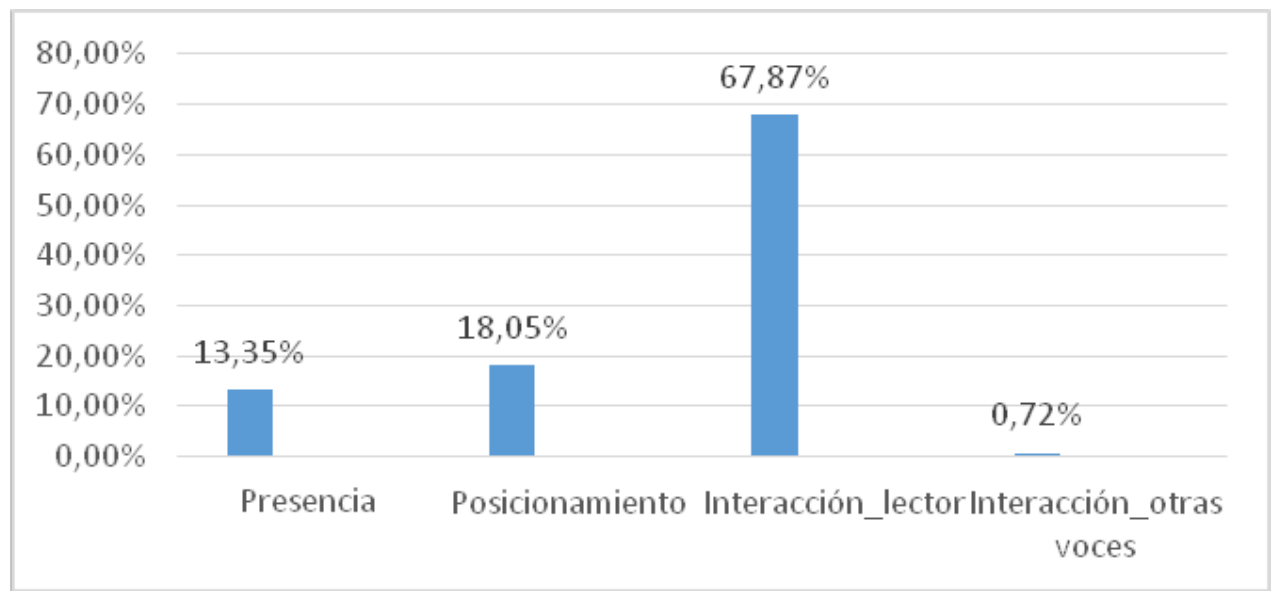

Figura 2. Dimensiones que se usan en el corpus según su recurrencia

Tal y como se observa en el Gráfico 1, la dimensión que alcanza una frecuencia más alta es la interacción con el lector con el porcentaje del $67,87 \%$. Menos frecuentemente, se utilizan recursos para posicionarse respecto a los contenidos con el 18,05\% o para que el autor se presente explícitamente en el texto con el 13,35\%. Los casos en los que se producen interacciones con otras voces, con el $0,72 \%$, son un resto.

Estos resultados iniciales requieren un estudio más pormenorizado de lo que sucede dentro de cada dimensión en términos de recursos y expresiones concretas utilizadas.

\section{a) Autorreferencia}

En esta dimensión de análisis se identifican los recursos que utiliza el autor para situarse dentro del texto de manera explícita; es decir, para distinguir su propia voz de aquello que él no expresa. Dentro de esta, se ha constatado la presencia de un total de cuatro recursos diferentes que se reflejan en la siguiente tabla junto con las expresiones utilizadas:

Tabla 1. Recursos y expresiones para la autorreferencia en el corpus

\begin{tabular}{|l|c|}
\hline \multicolumn{2}{|c|}{ Presencia del autor en el texto } \\
\hline $\begin{array}{l}\mathbf{1}^{\mathbf{a}} \text { persona del singular (desinencia del verbo) } \\
\text { (Decir, creer, recomendar, nombrar, "exponer, advertir, pensar, mencionar, referir, } \\
\text { pensar y encontrar) }\end{array}$ & $\mathbf{8 3 , 8 \%}$ \\
\hline $\begin{array}{l}\text { Adjetivo posesivo en } \mathbf{1}^{\mathbf{a}} \text { persona del singular } \\
(\mathrm{Mi})\end{array}$ & $\mathbf{8 , \mathbf { 1 } \%}$ \\
\hline $\begin{array}{l}\mathbf{1}^{\mathbf{a}} \text { persona del plural de modestia (desinencia del verbo) } \\
(\text { Decir, creer) }\end{array}$ & $\mathbf{5 , 4 \%}$ \\
\hline $\begin{array}{l}\text { Pronombre personal en } \mathbf{1}^{\mathbf{a}} \text { persona del singular } \\
\left(Y_{O}\right)\end{array}$ & $\mathbf{2 , 7 \%}$ \\
\hline
\end{tabular}


Como se observa en la Tabla 2, los informantes utilizan con más frecuencia la primera persona del singular en la desinencia del verbo en el 83,8\%, fundamentalmente con verbos como "decir", "creer" y "recomendar", que son aquellos que se repiten con más frecuencia. Los demás verbos aparecen en una sola ocasión con la desinencia en primera persona del singular. Algunos ejemplos ilustrativos de este recurso:

(1) Creo que puede ser de gran utilidad sobre todo para estudiantes de secundaria que necesiten refuerzo con la gramática, ya que dispone de muchos ejemplos y ejercicios con sus respectivas soluciones.

Informante 1.7

Ocasionalmente, los informantes utilizan adjetivos posesivos en primera persona del singular en el 8,1\% de los casos. Estos son los únicos casos que aparecen:

(2) En mi opinión el libro está bien organizado, ya que hay apartados separados en subapartados que son de gran ayuda.

Informante 1.3

La $1^{\text {a }}$ persona del plural de modestia como desinencia del verbo con los verbos "decir" y "creer" aparece en una única ocasión en cada caso:

(3) Como bien hemos dicho antes, es muy fácil de usar, ya que facilita una organización en forma de la ficha y también un índice temático.

Informante 1.4

Finalmente, de manera residual, en un único caso, se observa el uso del pronombre personal en primera persona del singular:

(4) Yo creo que nadie.

Informante 2.37

Los recursos y expresiones empleados para autorreferenciarse y el porcentaje de frecuencia de cada uno de estos constatan que no se utilizan variedad ni de recursos ni de expresiones y, por ende, que carecen de un léxico variado.

\section{b) Posicionamiento del autor respecto a los contenidos}

Esta otra dimensión de análisis se concreta en el corpus en tres categorías con diferentes propósitos: mitigadores (el 70\%), recursos que se utilizan para eludir el compromiso del autor; intensificadores (el 20\%), recursos que sirven para intensificar o enfatizar las ideas del autor; y marcadores de actitud (el 10\%), recursos que marcan la actitud (acuerdo o desacuerdo, necesidad, importancia) del autor. En la siguiente tabla, se recogen los recursos y expresiones que se utilizan para cada uno de estos: 
Tabla 2. Recursos y expresiones para posicionarse respecto a los contenidos en el corpus

\begin{tabular}{|l|l|c|}
\hline \multicolumn{2}{|c|}{ Posicionamiento del autor respecto a los contenidos } & $\mathbf{5 7 , 1 4 \%}$ \\
\hline Mitigadores & $\begin{array}{l}\text { Perífrasis con "poder" } \\
\text { (Decir, interesar, ser, valer, utilizar, aclarar, comparar, olvidar) } \\
\text { Verbos en tiempo condicional } \\
\text { (Perífrasis verbales con "poder") } \\
\text { Adverbios que expresan posibilidad } \\
\text { (Posiblemente) }\end{array}$ & $\mathbf{4 0 \%}$ \\
\hline Intensificadores & $\begin{array}{l}\text { Adverbios de certeza } \\
\text { (Claramente, totalmente, obviamente, definitivamente) } \\
\text { Expresiones de certeza } \\
\text { (Sin duda, sin duda alguna) }\end{array}$ & $\mathbf{6 0 \%}$ \\
\hline $\begin{array}{l}\text { Marcadores de } \\
\text { actitud }\end{array}$ & $\begin{array}{l}\text { Adverbios de tiempo } \\
\text { (Habitualmente) } \\
\text { Perífrasis verbales para destacar información } \\
\text { (Es necesario comentar, es imprescindible destacar, es impor- } \\
\text { tante reseñar) } \\
\text { Verbos de advertencia } \\
\text { (Advertir) }\end{array}$ & $\mathbf{6 0 \%}$ \\
\hline
\end{tabular}

En el caso de los mitigadores, los recursos que más se utilizan son las perífrasis verbales con "poder" que implican posibilidad, destacando los verbos "decir", "interesar" y "ser". Algunos ejemplos ilustrativos:

(5) Este libro podría ser muy útil para estudiantes de carreras que tengan relación con el lenguaje así como estudiantes de Lenguas Modernas o de alguna Filología.

Informante 1.34

Los tiempos verbales en condicional son el siguiente recurso más frecuente con expresiones de perífrasis verbales con "poder". Por ejemplo:

(6) Este libro puede ser de gran utilidad si estas [sic] estudiando una carrera de humanidades, ya que los temas que se presentan en él le dan mucha información sobre ellos y podrían utilizarse en diferentes áreas de la lingüística.

Informante 1.31

De manera residual, aparece únicamente el adverbio "posiblemente" que indica posibilidad:

(7) En definitiva, la gramática didáctica del español, presenta muchos aspectos de novedad que lo hace, sin duda, más atractivo porque posiblemente el lector se vea obligado a discrepar o a discutir sobre algunos fenómenos concretos. 
Respecto a los recursos utilizados para intensificar el compromiso, los estudiantes, mayoritariamente, usan adverbios de certeza, entre los cuales destaca "claramente". Algunos ejemplos ilustrativos:

(8) Por todo lo mencionado anteriormente, es un libro totalmente recomendable, con el que aquel que quiera dominar la gramática de esta lengua podrá contar.

Informante 1.14

(9) Libro de reciente incorporación a nuestro catálogo claramente dirigido al lector que se quiere iniciar en la gramática española.

Informante 2.46

En cuanto a las expresiones de certeza con el 40\% de recurrencia, se ha registrado únicamente la siguiente expresión: "sin duda" (alguna). Ejemplo:

(10) Éste [sic] es, sin duda, un libro que puede interesar especialmente a estudiantes de prácticamente cualquier grado académico y profesores de niveles no universitarios.

Informante 2.47

Respecto a los recursos y expresiones que se utilizan para marcar la actitud del autor, cabe decir que los informantes tienden a utilizar perífrasis verbales que sirven para destacar la información (el 60\%). Algunos ejemplos ilustrativos:

(11) Es necesario comentar, las soluciones que Leonardo ha decidido poner al final del libro.

Informante 1.15

(12) Por último es imprescindible destacar los numerosos ejemplos e incorporaciones de análisis sintácticos [sic] que ofrece, todo esto junto con ejercicios y sus respectivas soluciones para fomentar la práctica de lo aprendido.

Informante 1.17

Para los adverbios de tiempo (el 20\%) se constata únicamente "habitualmente":

(13) Habitualmente usado por estudiantes de grados de Lenguas o algún doble grado que requiéra [sic] esta asignatura.

Informante 1.15

Por último, solo se utiliza el verbo de advertencia "advertir" en el 20\% de los casos:

(14) Advierto que es posible que el alumnado de primaria no este [sic] preparado para este contenido.

Informante 1.25

En resumen, los resultados anteriores constatan el predominio de la mitigación del compromiso por parte del autor tanto por ser la categoría más frecuente como por ser 
la más variada a nivel de recursos y expresiones. Aun así, en términos generales, los recursos y expresiones que se utilizan en esta dimensión, al igual que en la anterior, se sitúan en niveles bajos de variedad.

C)

Interacción con el lector

Esta dimensión de análisis se refiere a los recursos y expresiones que hacen posible que el autor del texto interactúe con el posible lector, haciendo que se implique. En la siguiente tabla, se recogen los recursos y expresiones que han servido precisamente a este propósito.

Tabla 3. Recursos y expresiones para interactuar con el lector en el corpus

\begin{tabular}{|l|r|}
\hline \multicolumn{2}{|c|}{ Interacción con el lector } \\
\hline $\begin{array}{l}\mathbf{1}^{\mathbf{a}} \text { personal del plural inclusivo (desinencia del verbo) } \\
\text { (Encontrar, buscar, decir, estar, entender, acceder, estudiar, dar, observar, ver, utilizar, } \\
\text { presentar, hacer, creer, "comparar", introducir, considerar, conseguir y recurrir) }\end{array}$ & $\mathbf{5 0 , 6} \%$ \\
\hline $\begin{array}{l}\mathbf{1}^{\mathbf{a}} \text { personal del plural inclusivo (pronombre) } \\
\text { (Ante nosotros) }\end{array}$ & $\mathbf{0 , 5 3 \%}$ \\
\hline $\begin{array}{l}\mathbf{2}^{\mathbf{a}} \text { persona del singular (desinencia del verbo) } \\
\text { (Encontrar, buscar, ayudar, practicar) }\end{array}$ & $\mathbf{3 , 2} \%$ \\
\hline $\begin{array}{l}\text { Adjetivo posesivo en } \mathbf{1}^{\mathbf{a}} \text { persona del plural } \\
\text { (Nuestra lengua) }\end{array}$ & $\mathbf{6 , 9 1 \%}$ \\
\hline $\begin{array}{l}\text { Pronombre (no sujeto) en } \mathbf{1}^{\mathbf{a}} \text { persona del plural } \\
\text { (Nos + ayudar, hablar, ofrecer, facilitar, explicar, encontrar, buscar, estudiar, prac- } \\
\text { ticar, surgir, presentar, hacer, comentar, resolver, dar, resultar, concretar, servir, in- } \\
\text { troducir, informar, enseñar, aclarar, orientar y proporcionar) }\end{array}$ & $\mathbf{3 5 , 6 \%}$ \\
\hline $\begin{array}{l}\text { Pronombre (no sujeto) en } \mathbf{2}^{\mathbf{a}} \text { persona del singular } \\
\text { (Te + ayudar, facilitar, recomendar) }\end{array}$ & $\mathbf{2 , 1} \%$ \\
\hline $\begin{array}{l}\text { Pronombre (no sujeto) en } \mathbf{2}^{\mathbf{a}} \text { persona del plural } \\
\text { (Os + presentar) }\end{array}$ & $\mathbf{1 , 0 6 \%}$ \\
\hline
\end{tabular}

Para interactuar con el lector, se produce un despliegue abundante de recursos entre los que destaca el uso de la primera persona del plural inclusivo como desinencia del verbo. Para ello destaca el uso del verbo "encontrar" y los verbos "buscar" y "decir"; los demás se utilizan en una única ocasión. Algunos ejemplos ilustrativos:

(15) Echando un vistazo al índice temático podremos llegar fácilmente a la cuestión que buscamos.

Informante 1.10

Además de este recurso, también es bastante frecuente, con un porcentaje del $35,1 \%$, el uso del pronombre (no sujeto) en $1^{a}$ persona del plural. En este caso, destacan por su uso repetido: "nos" + "ayudar", "hablar", "ofrecer", "facilitar" y "explicar". Algunos ejemplos: 
(16) Por otra parte, nos ofrece un índice temático muy detallado, con un diseño claro y riguroso.

Informante 1.24

Los demás recursos observados son de una frecuencia muy inferior que, sin alcanzar el $10 \%$, pueden considerarse un resto. Entre estos, el uso de la $1^{\text {a }}$ persona del plural inclusivo como pronombre en una única ocasión:

(17) El libro que tenemos ante nosotros, es muy buen manual de la gramática didáctica del español.

Informante 4.74

En el caso de la $2^{\text {a }}$ persona del singular como desinencia del verbo, se observa un uso muy limitado de los verbos que aparecen en la tabla. Algunos casos:

(18) Para terminar encontraras [sic] las soluciones a los ejercicios que se plantean en cada capítulo y, además, hay un indice [sic] temático.

Informante 1.1

(19) Al final encontraras [sic] las soluciones a los ejercicios así como un índice temático, que te ayudará a manejarte con el libro.

Informante 1.23

Para el adjetivo posesivo en $1^{\text {a }}$ persona del plural solo se usa: "nuestra" + "lengua".

(20) Hay que ir más allá para tener un perfecto manejo sobre la gramática ya sea de nuestra lengua materna o de otra lengua que se estudie.

Informante 2.52

En el caso del pronombre no sujeto en $2^{\text {a }}$ persona del singular se usan únicamente las expresiones de la tabla en una única ocasión cada una:

(21) Al final encontraras [sic] las soluciones a los ejercicios así como un índice temático, que te ayudará a manejarte con el libro.

Informante 1.23

(22) Te facilita explicaciones claras organizadas en forma de ficha con múltiples ejemplos, ejercicios resueltos, todos ellos recogidos en un índice temático al principio del libro.

Informante 1.31

Por último, para el pronombre no sujeto en $2^{\mathrm{a}}$ persona del plural solo aparece: "os" + "presentar". Por ejemplo:

(23) El libro os presenta cinco capítulos de los cuáles [sic], el primero nos hace una breve introducción sobre lo que vamos a estudiar a continuación. 
En definitiva, el análisis de la interacción del autor con el lector revela que, aunque aparece una amplia variedad de recursos, solo destaca el uso de dos de ellos y el resto son de uso prácticamente residual. Nuevamente, en el análisis de los recursos empleados evidencia una escasez de expresiones y, por tanto, de vocabulario de los informantes.

d)

\section{Interacción con otras voces}

La interacción con otras voces se refiere a las ideas que el autor del texto extrae de otros textos para comparar, contrastar y discutir el conocimiento. Estas ideas tendrían que estar muy bien diferenciadas de las ideas del autor. En este caso, solo presenta un recurso: la cita indirecta. Para ello, en los dos únicos casos que aparecen, se utilizan las expresiones "según X" o "de acuerdo con X". Por ejemplo:

(24) Según él el libro resuelve dudas gramaticales y presenta explicaciones claras con múltiples ejemplos

Informante 1.25

(25) De acuerdo a lo mencionado anteriormente por el autor, el libro estará dividido en secciones, partiendo desde las clases de palabras (sustantivos, adjetivos, determinativos, pronombres, verbos, adverbios, preposiciones, conjunciones y [sic] interjecciones), deteniéndose [sic] posteriormente en las oraciones y grupos, a continuación haciendo énfasis en la fonética y fonología y concluyendo con la ortografía.

Informante 2.44

Es necesario señalar que estas citas no siguen ningún sistema de citación para que el lector localice las fuentes, ni se refieren de manera explícita al autor que están citando. Así, además de ser residual la interacción con otras voces, se manifiesta desconocimiento por los sistemas de citación.

\section{Corpus parciales}

\section{Primer curso}

El estudio pormenorizado de cada una de las dimensiones de análisis resuelve que los estudiantes de este curso no gestionan convenientemente los recursos para la construcción de la voz en el texto académico. Esto se observa en el uso constatado de las expresiones tanto para autorreferenciarse como para posicionarse ante el contenido o para interactuar con el lector o con otras voces.

La autorreferencia, con un porcentaje de uso del 16,6\%, se lleva a cabo fundamentalmente a través de la desinencia verbal en primera persona del singular, empleo que contraviene los convencionalismos y especificidades del discurso académico en lengua española. Solo en dos ocasiones se constata la presencia de la primera persona del plural de modestia, uso instaurado en la comunidad discursiva a la que pertenece el corpus estudiado.

Por otra parte, en relación al uso de expresiones para posicionarse ante el contenido, se observa que un buen número de informantes las utilizan (el 36,6\%). 
Ahora bien, tales expresiones tienen casi siempre (el 74,3\% de los casos) un efecto mitigador del nivel de compromiso con aquello que se dice. Se presentan las ideas como posibilidad y no tanto como hechos verificados. Otro tipo de posicionamientos como el de enfatizar las proposiciones o marcar la actitud del autor son muy escasos. De ello, parece desprenderse que los informantes no elaboran reseñas que desempeñen funciones como la de analizar críticamente una obra y expresar juicios de valor.

En relación a las expresiones para interactuar con lector, cuyo empleo alcanza el $45 \%$, se constata un uso mayoritario ajustado a las convenciones del discurso académico. No obstante, se dan casos que contravienen ese uso como es el empleo del pronombre personal "tú" o "vosotros" y el pronombre no sujeto "te".

Por último, cabe señalar que solo se da un caso de interacción con otras voces, lo cual es esperable teniendo en cuenta el contexto de producción del texto. Conviene mencionar que este caso no sigue ningún procedimiento convencional de citación.

\section{Segundo curso}

El análisis del corpus de este curso confirma que los estudiantes tampoco gestionan convenientemente los recursos para la construcción de la voz en el texto académico, lo cual se ha podido observar en las cuatro dimensiones de análisis de este estudio.

Para empezar, la autorreferencia se presenta de manera ocasional en los textos de este curso con un porcentaje del 21,6\%. Sin embargo, ninguno de los recursos que se utilizan para la autorreferencia son acordes a los convencionalismos del discurso académico español, siendo la primera persona del singular como desinencia del verbo y como pronombre personal los únicos que se han utilizado.

En cuanto a los recursos que sirven para que el autor se posicione respecto a los contenidos, una dimensión de análisis muy poco frecuente que apenas representa el $9,8 \%$ del corpus, se observa que las expresiones que se utilizan tienen casi siempre un carácter mitigador, de elusión del compromiso ocultándose tras la posibilidad. Apenas se observan casos de intensificación de las ideas y estos siempre marcan la necesidad. Por consiguiente, no producen un texto en el que se expresen juicios de valor o se analice e interprete de manera crítica la obra objeto de la reseña.

Respecto a las expresiones para interactuar con lector, a las cuales recurre el $67 \%$ del corpus, se constata que los informantes se ajustan a las convenciones del discurso académico español mediante el uso de la primera persona del plural inclusivo como desinencia del verbo, pronombre no sujeto o adjetivo posesivo. Ahora bien, también en este curso, aunque de manera aislada, se dan algunos casos que contravienen esos convencionalismos a través del empleo del pronombre "tú".

Finalmente, nuevamente se da un solo caso de interacción con otras voces, lo cual, como ya se menciona previamente, es esperable por las características del contexto de producción del texto. Sí cabe destacar que, al igual que en el primer curso, este caso no sigue ningún procedimiento convencional de citación. 


\section{Tercer curso}

Los resultados derivados del análisis del corpus del tercer curso constatan que los estudiantes configuran una voz de autor que se orienta, fundamentalmente, a la interacción con el lector. Esto se extrae del $97 \%$ de los recursos orientados a la interacción con el lector observados en estas reseñas, mediante el empleo de la primera persona del plural inclusivo en la desinencia del verbo, el pronombre no sujeto y el adjetivo posesivo.

El 3\% se atribuye a recursos para posicionarse respecto a los contenidos. Todos ellos utilizados para mitigar la voz del autor mediante la expresión de la posibilidad. Esto resulta en una voz muy poco convincente, apenas persuasiva para el lector, que no expresa auténticos juicios de valor.

\section{Cuarto curso}

El análisis de las reseñas de este curso también señala hacia un autor que construye su voz orientada, fundamentalmente, a la interacción con el lector, lo que queda representado en el $87 \%$ de los recursos y expresiones utilizadas. Esta interacción, aunque resulte poco variada en cuanto a las expresiones, se realiza de acuerdo con las especificidades del lenguaje académico español.

De una frecuencia del 13\% restante, los informantes de este curso utilizan recursos y expresiones para posicionarse respecto a los contenidos. Entre estos, destacan las expresiones de certeza para intensificar el compromiso y también se recurrirá a la posibilidad para eludir ese compromiso. En ambos casos, estos posicionamientos resultan insuficientes para la expresión de juicios de valor y el análisis crítico de la obra reseñada.

\section{Conclusión}

En este estudio se ha analizado cómo construyen la voz de autor los estudiantes de una titulación de grado y qué características tiene esa trayectoria. De este análisis se extraen importantes conclusiones sobre la manera que tienen estos autores de autorreferenciarse, posicionarse respecto a los contenidos, interactuar con el lector y con otras voces.

Por una parte, en cuanto a la autorreferencia, se ha observado que los estudiantes de los primeros cursos, al utilizar la primera persona del singular, se materializan en las reseñas de una manera que no se ajusta a los convencionalismos y especificidades del contexto social en el que tienen lugar sus producciones (Goethals y Delbecque, 2001). Este modo de presentarse en el texto se diluye a medida que pasan los cursos hasta desaparecer por completo en el tercer curso, ocultándose tras la tercera persona del singular. Este tipo de autor cada vez más neutro, más tenue, viene a coincidir con lo que han observado otros autores (Castro y Sánchez, 2013; Aguilar y Fregoso, 2013).

Por otra parte, en cuanto a la posición que adopta el autor con respecto a lo que escribe, el análisis constata que los informantes llevan a cabo unos posicionamientos muy inconsistentes y poco complejos. Se prioriza el uso de recursos para mitigar el compromiso del autor expresando, sobre todo, posibilidad, y solo se marca la necesidad de los enunciados cuando se intensifica ese compromiso, no su importancia. 
Por lo tanto, como se ha visto en estudios similares (Venegas, Meza y Martínez, 2013; Gil-Salom y Soler-Monreal, 2014; Sánchez-Jiménez, 2017), los estudiantes no realizan un posicionamiento que les lleve a expresar auténticos juicios de valor o que denote que han realizado un análisis crítico.

A este respecto, según Castro y Sánchez (2013), los estudiantes de los primeros cursos aún están en un período de transición entre los discursos sobre contenidos vitales que producían en niveles anteriores y el nuevo discurso sobre el conocimiento científico que deben producir en la universidad, por lo que cabe esperar que en sus textos aparezcan más valoraciones. Por el contrario, los estudiantes más experimentados en la comunidad discursiva, al intuir que la voz del experto está por encima de la voz del estudiante, ocultan sus propias valoraciones para garantizar el éxito de sus escritos.

Respecto a la interacción con el lector, se observa en todos los cursos, y cada vez con mayor énfasis, que los estudiantes tienen muy presente al lector del texto, pero esto no implica que configuren una voz de autor convincente o persuasiva. Por el contrario, llevan a cabo acercamientos al lector muy básicos y poco variados que inducen a considerar que los autores de este corpus de reseñas estén, quizás, mediatizados por la consigna, en la cual se enfatizaba la figura del lector. Alzari (2012), en un estudio sobre la reseña de formación en Historia, se encontró también con que los estudiantes utilizaban predominantemente la primera persona del plural como medio de interacción con el lector.

Por último, el hecho de que apenas se presenten interacciones con otras voces entra dentro de lo esperable teniendo en cuenta el contexto de producción de la tarea. En cambio, no deja de ser llamativo que los pocos informantes que introducen otras voces en el texto no siguen ningún sistema de citación y, por ende, no conjugan adecuadamente la voz del autor del texto con el conocimiento ajeno, lo cual es un conocimiento básico para producir un texto académico (López, 2016).

En definitiva, los resultados de este estudio confirman que los estudiantes de este corpus configuran una voz de autor totalmente neutra, en la cual se llevan a cabo relaciones de tipo muy primario con apenas variedad de recursos y de expresiones, lo cual ya no es suficiente en las nuevas programaciones de grado. Ahora, también se exige que el estudiante se posicione, que establezca un diálogo con la cultura disciplinar en la que se enmarca, con el lector y con el propio texto (Castelló, Corcelles, Iñesta, Bañales y Vega, 2011; Rodríguez y García, 2015).

Parece claro que la escritura como herramienta para comunicar el conocimiento científico no es algo que se desarrolla de manera natural, ni está garantizado por el paso de unos cursos a otros en la formación universitaria, por lo que es necesario que se lleven a cabo unidades didácticas donde se analicen los recursos para construir la voz de autor en textos académicos reales (Douglas, López y Padilla, 2011; Navarro, 2014).

\section{REFERENCIAS BIBLIOGRAFICAS}

Aguilar, L. E. y Fregoso, G. (2013). La lectura de la polifonía e intertextualidad en el texto científico. Revista Mexicana de Investigación Educativa, 18(57), 413-435. 
Alcaraz, M. A. y Salager-Meyer, F. (2005). Las reseñas de libros en español: estudio retórico y diacrónico. Spanish in Context, 2(1), 29-49. https://doi.org/10.1075/ sic.2.1.02ari.

Alzari, I. (2012). La reseña como género de formación en la carrera de Historia. $V$ Congreso Internacional de Letras (pp. 148-154). Buenos Aires: Facultad de Filosofía y Letras de la Universidad de Buenos Aires.

Alzari, I., D’Alessandro, J. y Radiminski, M. (2014). La reseña de formación en historia. En Navarro, F. (ed.), Manual de escritura para carreras de humanidades (pp. 287302). Buenos Aires: Facultad de Filosofía y Letras de la Universidad de Buenos Aires.

Babaii, E. y Ansary, H. (2007). A cross-disciplinary study of academic book reviews: defining a genre. En Pastukhov, A. (Ed.), Genres and text types in academic and media discourse (pp. 127-141). Oriol: Kartush Publisher.

Bazerman, C., Little, J., Bethel, L., Chavkin, T., Fouquette, D. y Garufis, J. (2005). Reference guide to writing across the curriculum. Indiana: Parlor Press y The WAC Clearinghouse.

Benveniste, E. (1974). Problemas de lingüística general I. México: Siglo XXI.

Brown, P. y Levinson, S. (1987). Politeness: Some Universals in Language Usage. Cambridge: Cambridge University Press.

Casamiglia, H. y Tusón, A. (2001). Las cosas del decir. Manual de análisis del discurso. Barcelona: Ariel.

Castelló, M., Corcelles, M., Iñesta, A., Bañales, G. y Vega, N. (2011). La voz del autor en la escritura académica: una propuesta para su análisis. Revista Signos, 44(76), 105-117. http://doi.org/10.4067/S0718-09342011000200001.

Castro de Castillo, E. (2005). La reseña. En Cubo de Severino, L. (ed.) Los textos de la ciencia. Córdoba (Argentina): Comunicarte.

Castro, M. C. y Sánchez, M. (2013). La expresión de opinión en textos académicos escritos por estudiantes universitarios. Revista Mexicana de Investigación Educativa, 18(57), 483-506.

Crismore, A. (1985). Metadiscourse in social studies texts. Urbana-Champaign: Universidad de Illinois.

Crismore, A., Markkanen, R. y Steffensen, M. (1993). Metadiscourse in persuasive writing: a study of texts written by American and Finnish university students. Written Communication, 10(1), 39-71. https://doi.org/10.1177/0741088393010001002.

Douglas, S., López, E. y Padilla, C. (2011). Argumentación y pensamiento crítico articulados en la alfabetización académica: reflexiones docentes. Los inicios de la alfabetización universitaria: el lugar de la lectura y la escritura (pp. 45-59). Buenos Aires: Universidad Tecnológica Nacional.

Giannoni, D. S. (2000). Hard words, soft technology, criticism and endorsement in the software review genre. En Gotti, M. Heller, D. y Dossena, M. (Eds.), Conflict and negotiation in specialized texts (pp. 335-363). Bern: Peter Lang. 
Giannoni, D. S. (2009). Negotiating research values across review genres: a case study in Applied Linguistics. En Hyland, K. y Diani, G. (Ed.), Academic evaluation. Review genres in university settings (pp. 17-33). Nueva York: Palgrave Macmillan.

Gil-Salom, L. y Soler-Monreal, C. (2014). Writers' positioning in literature reviews in English and Spanish computing doctoral theses. Journal of English for Academic Purposes, 16, 23-39.

Goethals, P. y Delbecque, N. (2001). Personas del discurso y "despersonalización". En Vázquez, G. (Ed.), Guía práctica del discurso académico escrito. ¿Cómo se escribe una monografía? (pp. 67-80) Madrid: Edinumen.

Gómez, L. (1998). Gramática didáctica del español. Madrid: SM.

Hyland, K. (2005). Metadiscourse. Nueva York: Contiuum.

Hyland, K. (2007). Disciplinary discourses: social interactions in academic writing. Michigan: Ann Arbor: University of Michigan Press.

López, E. (2016). Articulación entre saber ajeno y saber propio en escritos académicos estudiantiles de humanidades. Translaciones, Revista Iberoamericana de Lectura y Escritura, 3(6), 78-107.

Meza, P. (2017). El posicionamiento estratégico del autor en artículos de investigación: un modelo empíricamente fundado. Logos: Revista de Lingüística, Filosofía y Literatura, 27(1), 152-164. https://doi.org/10.15443/RL2711.

Motta-Roth, D. (1995). Book reviews and disciplinary discourse: defining a genre. Teachers of English to Speakers of Other Languages (TESOL) - 29th Annual Convention \& Exposition. Long Beach (California).

Motta-Roth, D. (2001). A construção social do gênero resenha acadêmica. Trabalhos Em Lingüística Aplicada, 38, 29-45.

Navarro, F. (2014). Gradación y compromiso en escritura académica estudiantil de Humanidades. Análisis contrastivo desde la teoría de la valoración. Estudios de Lingüística Aplicada, 32(60), 9-33.

Rodríguez, B. A. y García, L. B. (2015). Escritura de textos académicos: dificultades experimentadas por escritores noveles y sugerencias de apoyo. CPU-e, Revista de Investigación Educativa, 20, 251-265.

Salager-Meyer, F. (2001). 'This book portrays the worst form of mental terrorism': critical speech acts in medical English book reviews (1940-2000). En Kertész, A. (Ed.), Approaches to the pragmatics and scientific discourse (pp. 47-72). Bern: Peter Lang.

Sánchez-Jiménez, D. (2017). Implicaciones de la citación en la voz del autor en el discurso académico universitario: la memoria de máster escrita en español por estudiantes españoles y filipinos. Diálogo de la Lengua, VIII, 16-36.

Street, B. (1999). Academic Literacies. En Jones, C., Turner, J. y Street, B. (Eds.), Students writing in the university. Cultural and epistemological issues (pp.193-199). Amsterdam: John Benjamins Publishing Company . 
Tse, P. y Hyland, K. (2009). Discipline and gender: constructing rhetorical identity in book reviews. En Hyland, K. y Diani, G. (Eds.), Academic evaluation. Review genres in university settings (pp.105-121). Nueva York: Palgrave Macmillan.

Vande-Kopple, W. (1985). Some explorarotry discourse on metadiscourse. College Composition and Communication, 26(1), 82-93. https://doi.org/10.2307/357609.

Vande-Koople, W. (2012). The importance of studying metadiscourse. Applied Researh in English, 1(2), 37-44. https://doi.org/10.22108/ARE.2012.15453.

Venegas, R., Meza, P. y Martínez, J. M. (2013), Procedimientos discursivos en la atribución del conocimiento en tesis de lingüística y filosofía en dos niveles académicos. Revista de Lingüística Teórica y Aplicada, 51(1), 153-179.

\section{AneXo}

La Biblioteca de la UD quiere incorporar en su catálogo una reseña de los libros de reciente incorporación.

\section{¿Nos echas una mano?}

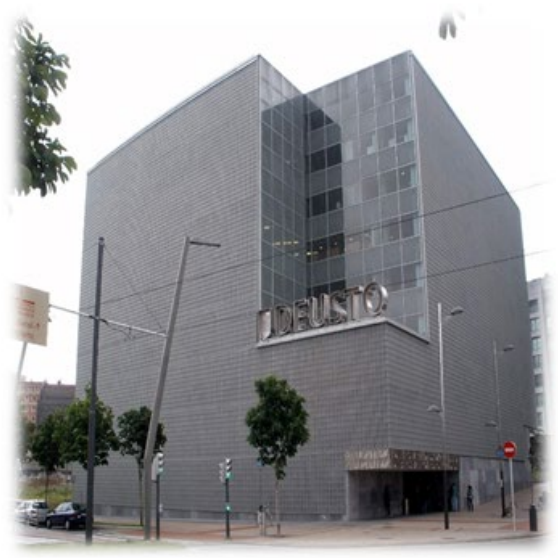

Necesitamos que escribas un texto de no menos de 200 palabras (máx. 300). TEN EN CUENTA QUE...

Este texto tiene como finalidad informar a los usuarios, estudiantes, que vayan a la biblioteca para saber si es el libro que están buscando.

Por ello, el texto que escribas debe incluir los aspectos del libro que consideres oportunos. La siguiente lista puede servir de ayuda: autor o autores, tema, organización del libro y contenidos, a quién puede interesar, valoración sobre su utilidad para la comunidad académica...

Nosotros te facilitaremos la portada, la contraportada, la presentación y el índice.

\section{¿COLABORA CON NOSOTROS!}

Figura 3. Instrumento definitivo de creación del corpus 